\title{
Intake of red and processed meat and risk of renal cell carcinoma: a meta-analysis of observational studies
}

\author{
Shaojing Zhang ${ }^{1}$, Qingwei Wang ${ }^{1}$ and Juanjuan $\mathrm{He}^{2}$ \\ ${ }^{1}$ Department of Urology Surgery, The First Affiliated Hospital, Zhengzhou University, Zhengzhou, Henan Province, China \\ ${ }^{2}$ Department of Breast Surgery, The First Affiliated Hospital, Zhengzhou University, Zhengzhou, Henan Province, China \\ Correspondence to: Juanjuan He, email: hejj1350@126.com
}

Keywords: red and processed meat, renal cell carcinoma, meta-analysis, relative risk

Received: November 01, 2016

Accepted: June 02, 2017

Published: June 16, 2017

Copyright: Zhang et al. This is an open-access article distributed under the terms of the Creative Commons Attribution License 3.0 (CC BY 3.0), which permits unrestricted use, distribution, and reproduction in any medium, provided the original author and source are credited.

\section{ABSTRACT}

Background: Findings on the association between intake of red and processed meat with renal cell carcinoma (RCC) risk are mixed. We conducted a meta-analysis to investigate this association.

Materials and Methods: Eligible studies up to August 31, 2016, were identified and retrieved by searching the MEDLINE and Embase databases along with manual review of the reference lists from the retrieved studies. The quality of the included studies was evaluated using the Newcastle-Ottawa Quality Assessment Scale. The summary relative risk (SRR) and corresponding $95 \%$ confidence interval (CI) were calculated using a random-effects model.

Results: Twenty-three publications were included in this meta-analysis: four cohort studies, one pooled study, and 18 case-control studies. The SRR (95\% CI) for the highest vs. lowest intake of red meat was $1.36(1.16-1.58$, Pheterogeneity < 0.001$)$; that for processed meat was $1.13(95 \%$ CI, 1.03-1.24, Pheterogeneity = 0.014). Linear dose-response analysis yielded similar results, i.e., the SRR for per $100 \mathrm{~g} /$ day increment of red meat and per $50 \mathrm{~g} /$ day increment of processed meat was 1.21 (95\% CI, 1.08-1.36) and 1.16 (95\% CI, 0.99-1.36), respectively. A non-linear association was observed only for red meat (Pnonlinearity $\mathbf{0 . 0 0 2}$ ), and not for processed meat (Pnonlinearity $=\mathbf{0 . 2 3 1}$ ). Statistically significant positive associations were observed for intake of beef, salami/ham/bacon/sausage, and hamburger.

Conclusions: This meta-analysis indicates a significant positive association between red and processed meat intake and RCC risk.

\section{INTRODUCTION}

In the United States, the incidence of kidney cancer is the seventh and tenth highest in men and women, respectively [1]. Renal cell carcinoma (RCC) is the most common malignancy of the kidney [2]. Globally, RCC incidence demonstrates regional variations, with agestandardized incidence rates being about 11.9 per 100,000 in developed areas and 2.5 per 100,000 in less developed regions [3]. The incidence of RCC has increased in most countries over the past decade [4]. However, the reasons for the regional and historical variations in RCC incidence are unknown. The demonstrated risk factors for RCC development include age, smoking [5], obesity
[6], hypertension [7], and acquired cystic kidney disease [8]. Although data are limited, a family history of kidney cancer [9], certain analgesics [10], history of diabetes [11], and occupational exposure (e.g., asbestos, silica, solder) have been linked to increased risk of RCC [12].

The consumption of red and processed meat has long been recognized as a risk factor of human cancer, as such meats are rich in well-established carcinogens, such as heterocyclic amines (HCAs), polycyclic aromatic hydrocarbons (PAHs), and $N$-nitroso compounds (NOCs) $[13,14]$. Many epidemiological studies have investigated the association between the consumption of red and processed meat and the risk of RCC [15-28]. Recently, two meta-analyses $[29,30]$ of observational studies have 
been published on this issue. According to the former meta-analysis of 13 case-control studies, Mohammed et al. [30] concluded that there is evidence supporting an independent relation between high consumption of red and processed meat and the incidence of kidney cancer. Whereas findings of the latter one [29], which included 12 case-control, 3 cohort and 1 pooled analysis, were not supportive of an independent relation between red or processedmeat intake and kidney cancer. Since then, numerous epidemiological studies [31-40] evaluating the aforementioned associations have been published and have reported inconsistent results. In addition, the exact form of the dose-risk relationship of these associations has not been clearly defined. To better understand this issue, we carried out a comprehensive meta-analysis of observational studies according to Meta-analysis Of Observational Studies in Epidemiology (MOOSE) guidelines [41].

\section{MATERIALS AND METHODS}

\section{Data sources and searches}

Two investigators (Z.S.J. and H.J.J.) conducted a computerized literature search independently in MEDLINE (from January 1, 1966) and Embase (from January 1, 1974) through to August 31, 2016. We searched the relevant studies using the following words and/or Medical Subject Heading (MeSH) terms: 1) intake OR consumption OR diet OR red meat OR processed meat OR preserved meat OR beef OR pork OR veal OR mutton OR lamb OR ham OR sausage OR bacon; 2) kidney OR renal; 3) carcinoma OR cancer OR neoplasm OR neoplasia; and 4) case-control OR cohort OR prospective OR retrospective. Furthermore, we reviewed the reference lists of the relevant articles to identify additional studies. Only studies published in English were included.

\section{Study selection}

In the present analysis, red meat was defined as beef, veal, pork, lamb, or a combination thereof [22]; processed meat was generally defined as meat products made largely from pork, veal, and beef that undergoes preservation such as curing, smoking, or drying [22]. We also assessed some specific red/processed meats, including beef, pork, hamburger, salami/ham/bacon/sausage, and barbecued/ pan-fried/broiled meat. We attempted to evaluate other subcategories that were described as "lamb" and "liver", but the number of included studies assessing these meats was too limited.

\section{Studies were included if they}

- were published as an original article;

- used a case-control or cohort design;

- reported relative risk (RR) estimates with corresponding 95\% CIs for the association between red and/or processed meat intake and the risk of RCC.

Non-peer-reviewed articles, abstracts, commentaries/ letters, ecologic assessments, correlation studies, experimental animal studies, and mechanistic studies were excluded. When multiple reports on the same study were available, only the most informative one was considered.

\section{Data collection and items}

A standardized data collection sheet was designed before the extraction. Two investigators (Z.S.J. and H.J.J.) separately extracted the basic information (first author's last name, location, publication year, sample source, duration of follow-up, number of cases and non-cases), data of interest (methods of ascertainment of dietary variables, exposure type [total or individual meats], comparison groups, methods of outcome assessment, RR [95\% CI] for the highest vs. lowest level), and adjustments. From each study, we extracted the risk estimates that reflected the greatest degree of control for potential confounders.

\section{Quality assessment of individual studies}

We used the NOS checklist to assess study quality [42], where the quality of case-control and cohort studies is assessed using three parameters: selection (four items, each awarded one star), comparability (one item, which can be awarded up to two stars), and exposure/outcome (three items, each awarded one star). A score of $\geq 7$ stars is indicative of a high-quality study.

\section{Statistical methods}

We used a random-effects model to calculate the SRRs (95\% CIs) for the high vs. low and dose-response analyses. This model accounts for heterogeneity among studies [43]. As outcomes were relatively rare, the ORs in the case-control studies were considered approximations of RRs. When sex-specific estimates were available, we analyzed for this separately. For studies [16, 18-20, 27 , $28,36,37,40]$ that presented results on meat subtypes separately, but not that for overall red/processed meat, we combined the results using a fixed-effects model, and then included the pooled RR estimates in the meta-analysis.

We used the $\chi^{2}$ test to assess heterogeneity among studies, defining significant heterogeneity as $P<0.10$. We also used the $\mathrm{I}^{2}$ statistic to explore the extent of inconsistency, with $\mathrm{I}^{2}>50 \%$ indicating high heterogeneity and $\mathrm{I}^{2}<25 \%$ indicating no significant heterogeneity [44]. We performed subgroup and meta-regression analysis on location, study design (case-control vs. cohort), FFQ type (validated vs. non-validated), available exposure data, study quality score, number of cases, and confounders (smoking status, BMI, dietary energy intake, alcohol consumption, intake of vegetables and fruits, history 
of hypertension). We conducted sensitivity analysis by repeating the meta-analysis of remaining studies after omitting one study at a time.

When possible, we performed linear dose-response meta-analysis per $100 \mathrm{~g}$ /day increment of red meat intake and per $50 \mathrm{~g}$ /day increment of processed meat intake using generalized least squares trend estimation (GLST) $[45,46]$. These methods require that the number of cases and person-time or controls for at least three quantitative exposure categories be known. GLST requires medians for categories of intake levels. For open-ended categories, we assumed that the range was the same as the adjacent interval. When the exposures were expressed as "times" or "servings", we converted it into grams (g) using $120 \mathrm{~g}$ and $50 \mathrm{~g}$ as a standard portion size for red meat and processed meat, respectively, as described in the WCRF/AICR report [22]. For the study [34] reporting intakes as g/1000 kcal/ day, the intake as $\mathrm{g} /$ day was estimated using the average energy intake reported in the article. We performed potential non-linear dose-response analysis using the bestfitting 2-term fractional polynomial regression model [47]. A likelihood ratio test was used to assess the difference between the non-linear and linear models to test for nonlinearity [47]. All statistical analyses were performed using R-package (Version 2.11.0 beta, R Development Core Team, NJ, USA) and Stata version 11.0 (StataCorp, College Station, TX, USA). A 2-sided test with $\alpha=0.05$ was used to indicate the level of significance.

\section{RESULTS}

\section{Search results and study characteristics}

The search strategy generated 2,211 citations, of which 59 were considered of potential value and for which the full text was retrieved for detailed evaluation. An additional seven articles were identified from a review of the references. Forty-three of these 66 articles were subsequently excluded from the meta-analysis. The studies by Di Maso et al. [48] and Bravi et al. [17] were based on the same data. We included the latter [17] because it had the most informative data. The studies by De Stefani et al. [23] and De Stefani et al. [33] were based on the same setting, but in different time periods, i.e., from 1988 to 1995 and from 1996 to 2004. Therefore, we included both studies. We also included two studies with overlapping reports $[19,35]$ : one on overall processed meat intake [35] and the other on red meat intake [19]. One pooled study included 13 independent cohorts [15]; another four cohort studies included four different cohorts (the European Prospective Investigation into Cancer and Nutrition study [EPIC] [32]; the NIH-AARP Diet and Health Study [34], the Japan Collaborative Cohort Study for Evaluation of Cancer Risk [JACC] Study [18], and California Seventhday Adventists [28]). An eventual total 23 publications were included in this meta-analysis (Figure 1).
The characteristics of these 23 publications are described in Tables 1 and 2. They comprised four prospective cohort studies [18, 28, 32, 34], one pooled study [15], and 18 case-control studies [16, 17, 20-27, $31,33,35,37-40]$. A total 14,285 patients with RCC and $1,821,615$ controls/participants were included. The studies were conducted in North America $(n=11)$, Europe $(n=7)$, Asia $(n=1)$, and South America $(n=3)$. The pooled study was conducted in the United States and in Europe. The methods used in all studies for assessing meat consumption were based on the food items semiquantitative Food Frequency Questionnaire (FFQ). The Newcastle-Ottawa Scale (NOS) scores ranged 5-9; 19 studies were deemed to be of high quality ( $\geq 7$ stars) (Supplementary Table 1).

\section{Red meat}

\section{High vs. low analysis}

Nineteen studies reported on the highest vs. lowest levels of red meat intake and RCC risk. The summary relative risk (SRR) was 1.36 (95\% confidence interval [CI], 1.16-1.58); there was evidence of high inter-study heterogeneity $\left(\mathrm{P}_{\text {heterogeneity }}<0.001, \mathrm{I}^{2}=71.3 \%\right.$; Figure $\left.2 \mathrm{~A}\right)$.

\section{Dose-response analysis}

Thirteen studies were included in the dose-response analysis of red meat intake and RCC risk. The SRR per $100 \mathrm{~g}$ /day increment was 1.21 (95\% CI, 1.08-1.36), with evidence of high heterogeneity $\left(\mathrm{P}_{\text {heterogeneity }}<0.001\right.$, $\mathrm{I}^{2}=73.6 \%$; Figure 2B). There was evidence of a nonlinear association of red meat intake and $\mathrm{RCC}$ risk $(P=0.002)$. Visual inspection of the curve suggested that the risk increased linearly up to approximately $240 \mathrm{~g} /$ day red meat intake. Above that, the risk increase became even steeper (Figure 2C).

\section{Processed meat}

\section{High vs. low analysis}

Nineteen studies reported on the highest vs. lowest level of processed meat intake and RCC risk. The SRR was 1.13 (95\% CI, 1.03-1.24), and there was evidence of moderate inter-study heterogeneity $\left(\mathrm{P}_{\text {heterogeneity }}=0.014\right.$, $\mathrm{I}^{2}=45.6 \%$; Figure $3 \mathrm{~A}$ ).

\section{Dose-response analysis}

Fourteen studies were included in the dose-response analysis of processed meat intake, and the SRR per $50 \mathrm{~g} /$ day increase was $1.16(95 \% \mathrm{CI}, 0.99-1.36)$, and there was high inter-study heterogeneity $\left(\mathrm{P}_{\text {heterogeneity }}<0.001\right.$, $\mathrm{I}^{2}=65.1 \%$; Figure $3 \mathrm{~B}$ ). There was no evidence of a nonlinear association between processed meat intake and RCC risk $(P=0.231$; Figure $3 \mathrm{C})$. 
Table 1: Characteristics of case-control studies of red and processed meat intake and renal cell carcinoma risk

\begin{tabular}{|c|c|c|c|c|c|c|c|}
\hline $\begin{array}{l}\text { Author/ year/ } \\
\text { Country }\end{array}$ & $\begin{array}{l}\text { number of } \\
\text { subjects } \\
\text { enrolled }\end{array}$ & $\begin{array}{c}\text { Outcome } \\
\text { determined }\end{array}$ & $\begin{array}{c}\text { Dietary } \\
\text { assessments }\end{array}$ & $\begin{array}{c}\text { Exposure } \\
\text { (Highest vs. lowest) }\end{array}$ & $\begin{array}{l}\text { RR }(95 \% \text { CI }) \\
\text { (Highest vs. } \\
\text { lowest) }\end{array}$ & Adjustments & Score \\
\hline \multicolumn{8}{|l|}{ Hospital-based } \\
\hline $\begin{array}{l}\text { Melkonian et al. } \\
2016, \text { USA [31] }\end{array}$ & $\begin{array}{l}659 \text { RCC cases } \\
699 \text { controls }\end{array}$ & Histological & $\begin{array}{l}\text { Self-administered } \\
\text { Validated FFQ }\end{array}$ & $\begin{array}{l}\text { Red meat } \\
\text { T3 vs. T1 }\end{array}$ & $2.28(1.67-3.10)$ & $\begin{array}{c}\text { Age, sex, BMI, history of } \\
\text { hypertension, smoking status, } \\
\text { total energy intake, total fruit } \\
\text { and vegetable intake }\end{array}$ & 7 \\
\hline $\begin{array}{l}\text { De Stefani et al. } \\
2012 \text { Uruguay } \\
\text { [33] }\end{array}$ & $\begin{array}{c}144 \mathrm{RCC} \\
2,532 \text { Controls }\end{array}$ & Histological & $\begin{array}{l}\text { Validated } \\
\text { FFQ-64 } \\
\text { Interview }\end{array}$ & $\begin{array}{l}\text { Processed meat: >28.3 vs. } \\
\qquad 11.4 \mathrm{~g} / \mathrm{d}\end{array}$ & $\begin{array}{l}1.21(0.65-2.25) \mathrm{M} \\
2.15(0.90-5.13) \mathrm{W}\end{array}$ & $\begin{array}{l}\text { Age, residence, BMI, } \\
\text { smoking status, smoking, } \\
\text { alcohol drinking, mate } \\
\text { consumption, total energy, } \\
\text { total vegetables and fruits, } \\
\text { total white meat }\end{array}$ & 7 \\
\hline $\begin{array}{l}\text { Aune et al. 2009, } \\
\text { Uruguay [40] }\end{array}$ & $\begin{array}{c}114 \text { RCC } \\
2,032 \text { Controls }\end{array}$ & Histological & $\begin{array}{l}\text { Validated } \\
\text { FFQ-64 } \\
\text { Interview }\end{array}$ & $\begin{array}{c}\text { Red meat: } 300.2 \text { vs. } 85.5 \mathrm{~g} / \mathrm{d} \\
\text { Beef: } 300 \text { vs. } 85.5 \mathrm{~g} / \mathrm{d} \\
\text { Lamb: } 150 \text { vs. } 0 \mathrm{~g} / \mathrm{d} \\
\text { Processed meat: }\end{array}$ & $\begin{array}{l}2.72(1.22-6.07) \\
2.53(1.14-5.59) \\
0.77(0.22-2.67) \\
1.23(0.68-2.22)\end{array}$ & $\begin{array}{c}\text { Age, sex, residence, } \\
\text { education, income, } \\
\text { interviewer, smoking status, } \\
\text { alcohol, dairy foods, grains, } \\
\text { fatty foods, fruits and } \\
\text { vegetables, fish, poultry, } \\
\text { mate drinking, BMI and } \\
\text { energy intake }\end{array}$ & 7 \\
\hline $\begin{array}{c}\text { Bravi et al. 2007, } \\
\text { Italy [17] }\end{array}$ & $\begin{array}{c}767 \mathrm{RCC} \\
1,534 \text { Controls }\end{array}$ & Histological & $\begin{array}{l}\text { Interview } \\
\text { FFQ-78 } \\
\text { validated }\end{array}$ & $\begin{array}{c}\text { Red meat: } 5.9 \text { vs. } 2.4 \text { serving/ } \\
\text { wk } \\
\text { Processed meat: } 3.9 \text { vs. } 0.9 \\
\text { serving/wk }\end{array}$ & $\begin{array}{l}0.84(0.62-1.14) \\
0.64(0.45-0.90)\end{array}$ & $\begin{array}{l}\text { Age, center, sex, period of } \\
\text { interview, education, } \\
\text { smoking, alcohol drinking, } \\
\text { BMI, family history of } \\
\text { kidney cancer, total energy } \\
\text { intake }\end{array}$ & 7 \\
\hline $\begin{array}{l}\text { Hsu et al. 2007, } \\
\text { Europe [16] }\end{array}$ & $\begin{array}{c}1,065 \mathrm{RCC} \\
1,509 \text { Controls }\end{array}$ & Histological & $\begin{array}{l}\text { Interview } \\
\text { FFQ-23 } \\
\text { validated }\end{array}$ & $\begin{array}{c}\text { Red meat: } \geq 1 \text { time } / \text { wk vs. }<1 \\
\text { time/month } \\
\text { Ham, salami, sausages } \geq 1 \\
\text { time/wk vs. }<1 \text { time/month }\end{array}$ & $\begin{array}{l}2.01(1.02-3.99) \\
1.03(0.71-1.51)\end{array}$ & $\begin{array}{l}\text { Age, country, sex, smoking, } \\
\text { education, } \\
\text { BMI, hypertension } \\
\text { medication use, alcohol } \\
\text { consumption, total white } \\
\text { meat consumption }\end{array}$ & 7 \\
\hline $\begin{array}{l}\text { Tavani et al. } 2000, \\
\text { Italy [21] }\end{array}$ & $\begin{array}{c}190 \text { RCC } \\
\text { 7,990 Controls }\end{array}$ & Histological & $\begin{array}{l}\text { Self-administered } \\
\text { FFQ, NA }\end{array}$ & $\begin{array}{c}\text { Red meat: }>6 \text { vs. } \leq 3 \\
\text { servings/wk }\end{array}$ & $1.1(0.8-1.6)$ & $\begin{array}{l}\text { Age, year of recruitment, } \\
\text { sex, education, smoking, } \\
\text { alcohol, fat, fruit and } \\
\text { vegetable intakes. }\end{array}$ & 5 \\
\hline $\begin{array}{l}\text { De Stefani et al. } \\
\text { 1998, Uruguay } \\
\text { [23] }\end{array}$ & $\begin{array}{c}121 \text { RCC } \\
243 \text { Controls }\end{array}$ & Histological & $\begin{array}{l}\text { Interview } \\
\text { FFQ-23 } \\
\text { validated }\end{array}$ & $\begin{array}{c}\text { Red meat: > } 365 \mathrm{vs} . \leq 208 \mathrm{~g} / \mathrm{d} \\
\text { Barbecued: }>53 \mathrm{vs} . \leq 12 \mathrm{~g} / \mathrm{d} \\
\text { Processed meat: }>53 \mathrm{vs} . \\
\leq 12 \mathrm{~g} / \mathrm{d}\end{array}$ & $\begin{array}{c}3.42(1.76-6.65) \\
2.071 .03-4.19 \\
0.78(0.45-1.39)\end{array}$ & $\begin{array}{l}\text { Age, sex, residence, urban- } \\
\text { rural status, education, BMI, } \\
\text { mate drinking. }\end{array}$ & 6 \\
\hline $\begin{array}{l}\text { Talamini et al. } \\
\text { 1990, Italy [26] }\end{array}$ & $\begin{array}{l}240 \text { RCC } \\
665 \text { Controls }\end{array}$ & Histological & $\begin{array}{l}\text { Interview } \\
\text { FFQ } \\
\text { NA }\end{array}$ & $\begin{aligned} \text { Salami: } & \geq 3 \text { serving/wk vs. } \\
& \text { the lowest }\end{aligned}$ & $\begin{array}{l}1.01(0.63-1.61) \\
1.25(0.85-1.85)\end{array}$ & $\begin{array}{l}\text { Age, sex, education, area of } \\
\text { residence, BMI }\end{array}$ & 5 \\
\hline \multicolumn{8}{|l|}{ Population-based } \\
\hline $\begin{array}{l}\text { Hu et al. 2011, } \\
\text { Canada [35] }\end{array}$ & $\begin{array}{c}1,345 \mathrm{RCC} \\
5,039 \text { Controls }\end{array}$ & Histological & $\begin{array}{l}\text { Validated } \\
\text { FFQ-69 } \\
\text { Interview }\end{array}$ & $\begin{array}{l}\text { Processed meat: } \geq 5.42 \\
\text { vs. } 0.94 \text { servings } / \mathrm{wk}\end{array}$ & $1.3(1.1-1.6)$ & $\begin{array}{l}\text { Age, province, education, } \\
\text { BMI, sex, alcohol use, } \\
\text { smoking, total vegetable and } \\
\text { fruit intake, and total energy } \\
\text { intake }\end{array}$ & 9 \\
\hline $\begin{array}{l}\text { Daniel et al. 2011, } \\
\text { USA [36] }\end{array}$ & $\begin{array}{c}1,192 \text { RCC } \\
1,175 \text { Controls }\end{array}$ & Histological & $\begin{array}{l}\text { Interviewer } \\
\text { Diet History } \\
\text { Questionnaire }\end{array}$ & $\begin{array}{c}\text { Red meat: } 42.0 \mathrm{vs} .11 .7 \\
\text { g/1000kal } / \mathrm{d} \\
\text { Barbecued meat: } 16.7 \mathrm{vs} .0 \\
\text { g/1000kal } / \mathrm{d} \\
\text { Pan-fried meat: } 15.6 \mathrm{vs} .0 .3 \\
\text { g/1000kal } / \mathrm{d} \\
\text { Broiled meat: } 7.6 \mathrm{vs} .0 \\
\text { g/1000kal } / \mathrm{d}\end{array}$ & $\begin{array}{l}1.11(0.83-1.48) \\
1.35(1.01-1.79) \\
1.05(0.80-1.38) \\
0.75(0.59-0.96)\end{array}$ & $\begin{array}{l}\text { Age, race, sex, education, } \\
\text { smoking status, BMI, } \\
\text { history of hypertension, } \\
\text { family history of cancer, } \\
\text { alcohol, intake of fruit and } \\
\text { vegetables, total energy } \\
\text { intake, and other meat intake } \\
\text { and/or cooking method } \\
\text { offsets }\end{array}$ & 8 \\
\hline $\begin{array}{l}\text { Brock et al. 2009, } \\
\text { USA [39] }\end{array}$ & $\begin{array}{c}323 \mathrm{RCC} \\
1,820 \text { Controls }\end{array}$ & Histological & $\begin{array}{l}\text { Self-administered } \\
\text { questionnaire } \\
\text { NA }\end{array}$ & $\begin{array}{c}\text { Red meat: }>1.7 \text { vs. } 0-0.8 \\
\text { servings } / \mathrm{d} \\
\text { Cured meat: }>0.6 \text { vs. } \\
0-0.1 \text { servings } / \mathrm{d}\end{array}$ & $\begin{array}{l}1.5(1 \cdot 0-2.4) \\
1.6(1 \cdot 1-2.5)\end{array}$ & $\begin{array}{l}\text { Age, sex, smoking, obesity, } \\
\text { hypertension, physical } \\
\text { activity, alcohol and } \\
\text { vegetable intake and } \\
\text { tea and coffee consumption }\end{array}$ & 9 \\
\hline $\begin{array}{l}\text { Grieb et al. 2009, } \\
\text { USA [37] }\end{array}$ & $\begin{array}{c}335 \text { RCC } \\
337 \text { Controls }\end{array}$ & Histological & $\begin{array}{l}\text { Interview } \\
\text { FFQ-70 } \\
\text { Validated }\end{array}$ & $\begin{array}{c}\text { Red meat: }>5 \text { vs. }<1 \text { time } / \text { wk } \\
\text { Bacon and sausage: }>5 \text { vs. } \\
<1 \text { time } / w k\end{array}$ & $\begin{array}{l}4.43(2.02-9.75) \\
1.28(0.63-2.62)\end{array}$ & $\begin{array}{l}\text { Age, sex, race, income, BMI, } \\
\text { smoking }\end{array}$ & 8 \\
\hline
\end{tabular}




\begin{tabular}{|c|c|c|c|c|c|c|c|}
\hline $\begin{array}{l}\text { Hu et al. 2003, } \\
\text { Canada [19] }\end{array}$ & $\begin{array}{c}1,279 \text { RCC } \\
5,380 \text { Controls }\end{array}$ & Histological & $\begin{array}{c}\text { Self-administered } \\
\text { FFQ-70 } \\
\text { Validated }\end{array}$ & $\begin{array}{c}\text { Beef, pork or lam:T3 vs.T1 } \\
\text { Hamburger:T3 vs.T1 } \\
\text { Bacon:T3 vs.T1 } \\
\text { Sausage:T3 vs.T1 }\end{array}$ & $\begin{array}{l}1.3(1.0-1.6) \\
1.4(1.1-1.8) \\
1.3(1.0-1.6) \\
1.5(1.2-2.0)\end{array}$ & $\begin{array}{c}\text { Age, sex, province, } \\
\text { education, BMI, alcohol use, } \\
\text { smoking and total energy }\end{array}$ & \\
\hline $\begin{array}{l}\text { Handa et al. 2002, } \\
\text { Canada [20] }\end{array}$ & $\begin{array}{c}461 \text { RCC } \\
672 \text { Controls }\end{array}$ & Histological & $\begin{array}{l}\text { Self-administered } \\
\text { FFQ-69 } \\
\text { NA }\end{array}$ & Beef: Q4 vs. Q1 & $1.2(0.7-2.0) \mathrm{M}$ & Age, smoking status, BMI & 7 \\
\hline $\begin{array}{c}\text { Yuan et al. 1998, } \\
\text { USA [22] }\end{array}$ & $\begin{array}{c}1204 \text { RCC } \\
1204 \text { Controls }\end{array}$ & Histological & $\begin{array}{l}\text { Interview } \\
\text { FFQ-40 } \\
\text { NA }\end{array}$ & Processed meat: Q4 vs. Q1 & $1.15(0.86-1.54)$ & $\begin{array}{l}\text { level of education, BMI, } \\
\text { history of hypertension } \\
\text { cigarettes, analgesics, use of } \\
\text { amphetamines }\end{array}$ & 7 \\
\hline $\begin{array}{l}\text { Wolk et al. } 1996 \text {, } \\
\text { multi centers [24] }\end{array}$ & $\begin{array}{c}1,185 \mathrm{RCC} \\
1,526 \text { Controls }\end{array}$ & Histological & $\begin{array}{l}\text { Self-administered } \\
\text { and interview } \\
\text { FFQ, NA }\end{array}$ & $\begin{array}{c}\text { Red meat: Q4 vs. Q1 } \\
\text { Processed meat: Q4 vs. Q1 }\end{array}$ & $\begin{array}{l}0.94(0.73-1.20) \\
0.94(0.73-1.22)\end{array}$ & $\begin{array}{l}\text { Age, sex, stud center, BMI, } \\
\text { smoking, total calories }\end{array}$ & 7 \\
\hline $\begin{array}{c}\text { Chow et al. 1994, } \\
\text { USA [25] }\end{array}$ & $\begin{array}{c}690 \text { RCC } \\
707 \text { Controls }\end{array}$ & Histological & $\begin{array}{l}\text { Self-administered } \\
\text { FFQ-65 } \\
\text { validated }\end{array}$ & $\begin{array}{c}\text { Red meat: }>9.3 \text { vs. } 4.3 \\
\text { servings } / \text { wk } \\
\text { Processed meat:5.0 vs. } 1.4 \\
\text { servings } / \text { wk }\end{array}$ & $\begin{array}{l}1.3(0.9-1.9) \\
1.0(0.7-1.5)\end{array}$ & $\begin{array}{l}\text { Age, sex, cigarette smoking, } \\
\text { and BMI. }\end{array}$ & 8 \\
\hline $\begin{array}{l}\text { Maclure et al. } \\
\text { 1990, USA [27] }\end{array}$ & $\begin{array}{c}203 \text { RCC } \\
604 \text { Controls }\end{array}$ & Histological & $\begin{array}{l}\text { Interview } \\
\quad \text { FFQ } \\
\text { Validated }\end{array}$ & $\begin{array}{c}\text { Beef: Q4 vs. Q1 } \\
\text { Pork: Q4 vs. Q1 } \\
\text { Bacon: Q4 vs. Q1 } \\
\text { Processed meat: Q4 vs. Q1 }\end{array}$ & $\begin{array}{c}3.4(1.6-7.2) \\
0.74(0.4-1.4) \\
0.85(0.47-1.5) \\
1.3(0.86-2.0)\end{array}$ & Age, sex & 7 \\
\hline
\end{tabular}

Abbreviation: BMI, body mass index; FFQ, food frequency questionnaire; NA, not available; M, men, W, women; RCC, renal cell carcinoma.

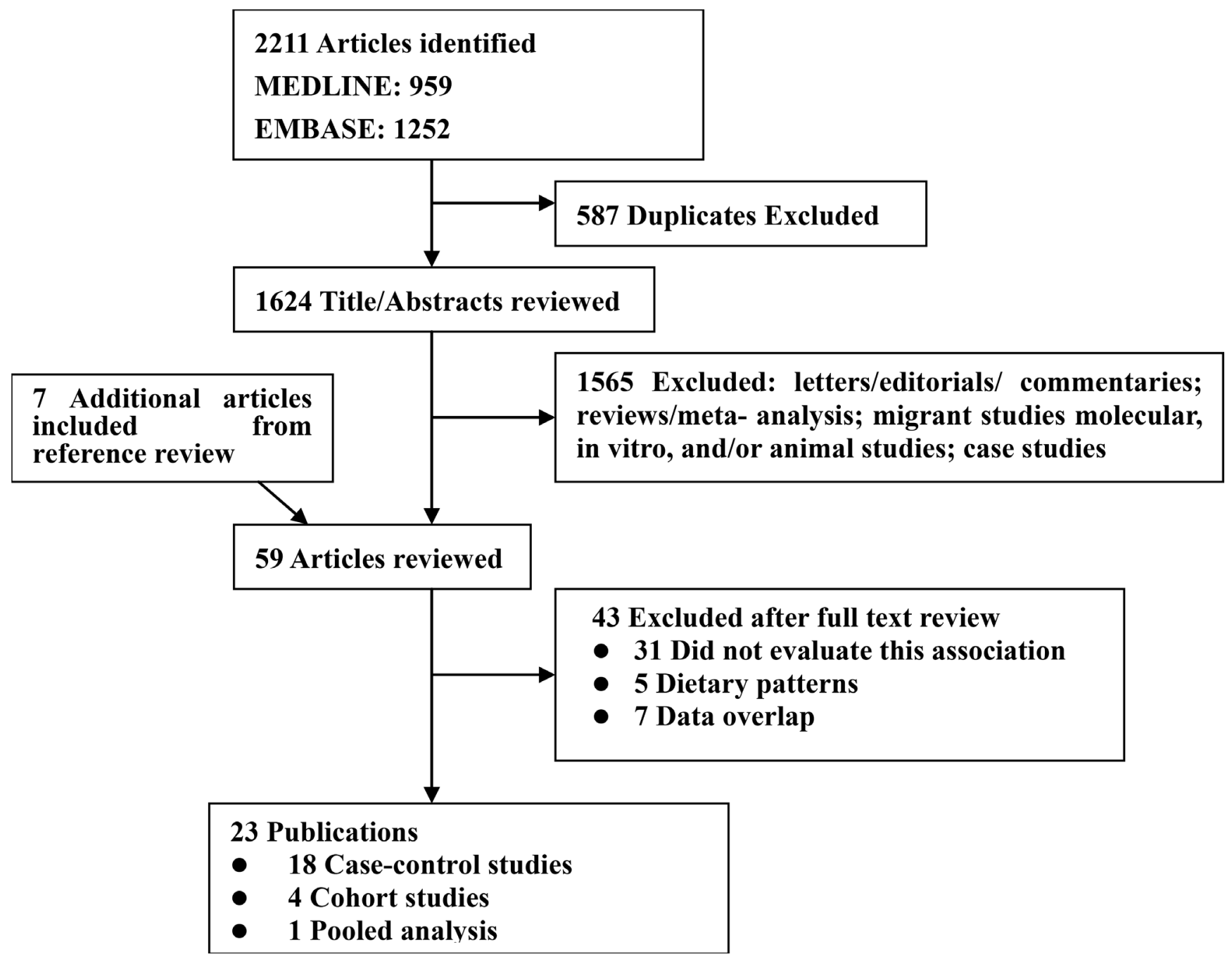

Figure 1: Flow diagram of systematic literature search on red and processed meat intake and renal cell carcinoma risk. 
Table 2: Characteristics of cohort studies of red and processed meat intake and renal cell carcinoma

\begin{tabular}{|c|c|c|c|c|c|c|c|}
\hline $\begin{array}{l}\text { Author/year, } \\
\text { Country }\end{array}$ & $\begin{array}{c}\text { Study name and } \\
\text { number of } \\
\text { subjects } \\
\text { FU, yr }\end{array}$ & $\begin{array}{c}\text { Case } \\
\text { ascertainment } \\
\text { Cases }(n)\end{array}$ & $\begin{array}{c}\text { Dietary } \\
\text { assessments }\end{array}$ & Exposure details & $\begin{array}{l}\text { RR }(95 \% \text { CI) } \\
\text { (Highest vs. } \\
\text { lowest) }\end{array}$ & Adjustments & Score \\
\hline $\begin{array}{l}\text { Rohrmann } \\
\text { et al. } 2015 \text { [32], } \\
\text { Europe }\end{array}$ & $\begin{array}{c}\text { EPIC } \\
N=375,851 \\
\text { FU, } 11.6 \mathrm{yr}\end{array}$ & $\begin{array}{l}\text { cancer or mortality } \\
\text { registries } \\
691 \text { RCC }\end{array}$ & $\begin{array}{l}\text { Self-administered } \\
\text { Validated } \\
\text { FFQ }\end{array}$ & $\begin{array}{c}\text { Red meat: }>80 \text { vs. } \\
0-9.9 \mathrm{~g} / \mathrm{d} \\
\text { Processed meat: }>80 \\
\text { vs. } 0-9.9 \mathrm{~g} / \mathrm{d}\end{array}$ & $\begin{array}{l}1.46(0.99-2.15) \\
1.23(0.84-1.79)\end{array}$ & $\begin{array}{c}\text { Age, center, sex, } \\
\text { education, BMI, history } \\
\text { of hypertension, smoking } \\
\text { status, duration of smoking, } \\
\text { energy intake,alcohol } \\
\text { consumption, fruit and } \\
\text { vegetable consumption }\end{array}$ & 9 \\
\hline $\begin{array}{l}\text { Daniel et al. } \\
\text { USA2012 [34] }\end{array}$ & $\begin{array}{c}\text { NIH-AARP Diet } \\
\text { and Health Study } \\
N=491,841 \\
\text { FU, } 9 \text { yr }\end{array}$ & $\begin{array}{c}\text { cancer registry } \\
1,816 \mathrm{RCC}\end{array}$ & $\begin{array}{c}\text { Self-administered } \\
\text { Validated } \\
\text { FFQ-124 }\end{array}$ & $\begin{array}{l}\text { Red meat: } 48.1 \text { vs. } \\
6.8 \mathrm{~g} / 1000 \mathrm{k} / \mathrm{d} \\
\text { Processed meat:19.9 } \\
\text { vs. } 1.4 \mathrm{~g} / 1000 \mathrm{k} / \mathrm{d}\end{array}$ & $\begin{array}{l}1.08(0.92-1.28) \\
1.12(0.95-1.32)\end{array}$ & $\begin{array}{l}\text { Age, sex, education, race, } \\
\text { marital status, family } \\
\text { history of any cancer, } \\
\text { BMI, smoking status, } \\
\text { hypertension, diabetes, } \\
\text { alcohol, total energy, } \\
\text { legumes, whole grains }\end{array}$ & 9 \\
\hline $\begin{array}{c}\text { Lee et al. 2008, } \\
\text { Europe and USA } \\
{[15]}\end{array}$ & $\begin{array}{c}13 \text { cohorts } \\
N=774,952 \\
\text { FU, } 7-20 \mathrm{yr}\end{array}$ & $\begin{array}{c}\text { medical records, } \\
\text { cancer registries } \\
1,478 \text { RCC }\end{array}$ & $\begin{array}{c}\text { Self-administered } \\
\text { Validated } \\
\text { FFQ }\end{array}$ & $\begin{array}{c}\text { Red meat: }>80 \text { vs. } \\
<20 \mathrm{~g} / \mathrm{d} \\
\text { Processed meat: } 12-27 \\
\text { vs. }<4 \mathrm{~g} / \mathrm{d}\end{array}$ & $\begin{array}{l}0.99(0.85-1.16) \\
1.06(0.88-1.28)\end{array}$ & $\begin{array}{c}\text { Age, history of } \\
\text { hypertension, BMI, } \\
\text { smoking, combination } \\
\text { of parity and age at first } \\
\text { birth, fruit } \\
\text { and vegetable } \\
\text { consumption, alcohol } \\
\text { intake, and total energy } \\
\text { intake }\end{array}$ & 9 \\
\hline $\begin{array}{c}\text { Washio et al. } \\
\text { 2005, Japan [18] }\end{array}$ & $\begin{array}{c}\text { JACC } \\
N=114,517 \\
\mathrm{FU}, 10 \mathrm{yr}\end{array}$ & $\begin{array}{c}\text { mortality registries } \\
\quad 48 \text { RCC }\end{array}$ & $\begin{array}{l}\text { Self-administered } \\
\text { Validated } \\
\text { questionnaire }\end{array}$ & $\begin{array}{c}\text { Beef: } 1-2 \text { vs. seldom } \\
\text { times/wk } \\
\text { Pork: } 1-2 \text { vs. seldom } \\
\text { times/wk } \\
\text { Ham and sausage: } 1-2 \\
\text { vs. seldom times/wk }\end{array}$ & $\begin{array}{l}1.73(0.74-4.08) \\
0.92(0.34-2.27) \\
1.16(0.42-3.24)\end{array}$ & Age, sex & 7 \\
\hline $\begin{array}{c}\text { Fraser et al. } \\
\text { 1990, USA [28] }\end{array}$ & $\begin{array}{c}\text { California } \\
\text { Seventh-day } \\
\text { Adventists } \\
N=34,198 \\
\text { FU, } 6.2 \text { yr }\end{array}$ & $\begin{array}{c}\text { mortality registries } \\
14 \text { RCC }\end{array}$ & $\begin{array}{l}\text { Self-administered } \\
\text { Validated } \\
\text { FFQ }\end{array}$ & $\begin{array}{c}\text { Beef: }>1 \text { vs. }<1 \\
\text { serving/wk }\end{array}$ & $1.59(0.49-5.01)$ & Age, sex & 6 \\
\hline
\end{tabular}

Abbreviation: EPIC, the European Prospective Investigation into Cancer and Nutrition; JACC, the Japan Collaborative Cohort Study for Evaluation of Cancer Risk Study; BMI, body mass index; FFQ, food frequency questionnaire; NA, not available, RCC, renal cell carcinoma; FU, follow-up.

\section{Subgroup, meta-regression, and sensitivity analyses}

Table 3 shows the results of the stratified and metaregression analyses. For high vs. low consumption of red meat, we observed an increased risk of RCC in case-control studies (SRR $=1.46 ; 95 \%$ CI, 1.18-1.81), but not in cohort studies $(\mathrm{SRR}=1.07 ; 95 \% \mathrm{CI}, 0.96-1.19)$. The SRRs were significant for studies conducted in North America $\left(\mathrm{SRR}=1.44 ; 95 \% \mathrm{CI}, 1.17-1.76 ; \mathrm{I}^{2}=68.6 \%\right)$ and South America $\left(\mathrm{SRR}=3.12 ; 95 \% \mathrm{CI}, 1.87-5.20 ; \mathrm{I}^{2}=72.3 \%\right)$, but not in those conducted in Europe (SRR $=1.04 ; 95 \%$ CI, $0.86-1.26$ ) and Asia (SRR $=1.30 ; 95 \%$ CI, 0.69-2.46). There was significant between-subgroup heterogeneity in stratified analysis of location $(P$ for difference $=0.038$ ). Adjustments by body mass index (BMI), smoking, history of hypertension, total energy intake, intake of vegetables and fruits, and alcohol consumption did not significantly change the SRR for RCC risk.

For high vs. low consumption of processed meat, we observed a borderline significant risk of RCC in both casecontrol $\left(\mathrm{SRR}=1.13 ; 95 \% \mathrm{CI}, 1.00-1.27 ; \mathrm{I}^{2}=55.4 \%\right)$ and cohort studies $\left(\mathrm{SRR}=1.11 ; 95 \% \mathrm{CI}, 0.99-1.25 ; \mathrm{I}^{2}=0\right)$. The SRR was significant for studies conducted in North America (SRR $=1.20 ; 95 \%$ CI, 1.07-1.34), but not for studies conducted in South America, Europe, and Asia.

In univariate meta-regression analysis, only location was a significant factor for the association between red meat intake and RCC risk; however, no variables were significant factors for processed meat intake.

The estimation of overall homogeneity and the effect of removing one study at a time from the analysis confirmed the stability of the relationship between intake of red and processed meat and RCC risk (data not shown). In addition, repeat analysis of high vs. low intake using the studies included in the linear dose-response analysis yielded results similar to that of the original analysis (red meat: $\mathrm{SRR}=1.20 ; 95 \% \mathrm{CI}, 1.07-1.34$; processed meat: $\mathrm{SRR}=1.13 ; 95 \% \mathrm{CI}, 1.00-1.27)$.

\section{Publication bias}

For intake of red meat, visual inspection of the funnel plot, as well as Egger's test $(P=0.087)$ and Begg's 
test $(P=0.005)$, indicated publication bias. The trim-andfill method indicated that eight additional risk estimates were needed to balance the funnel plot (Figure 4A), and the summary risk estimates were again not significant $(\mathrm{SRR}=1.09 ; 95 \% \mathrm{CI}, 0.92-1.29)$. For intake of processed meat, visual inspection of the funnel plot, as well as

\section{Red meat}

A

Study
ID

\section{Red meat}

B

Study
ID




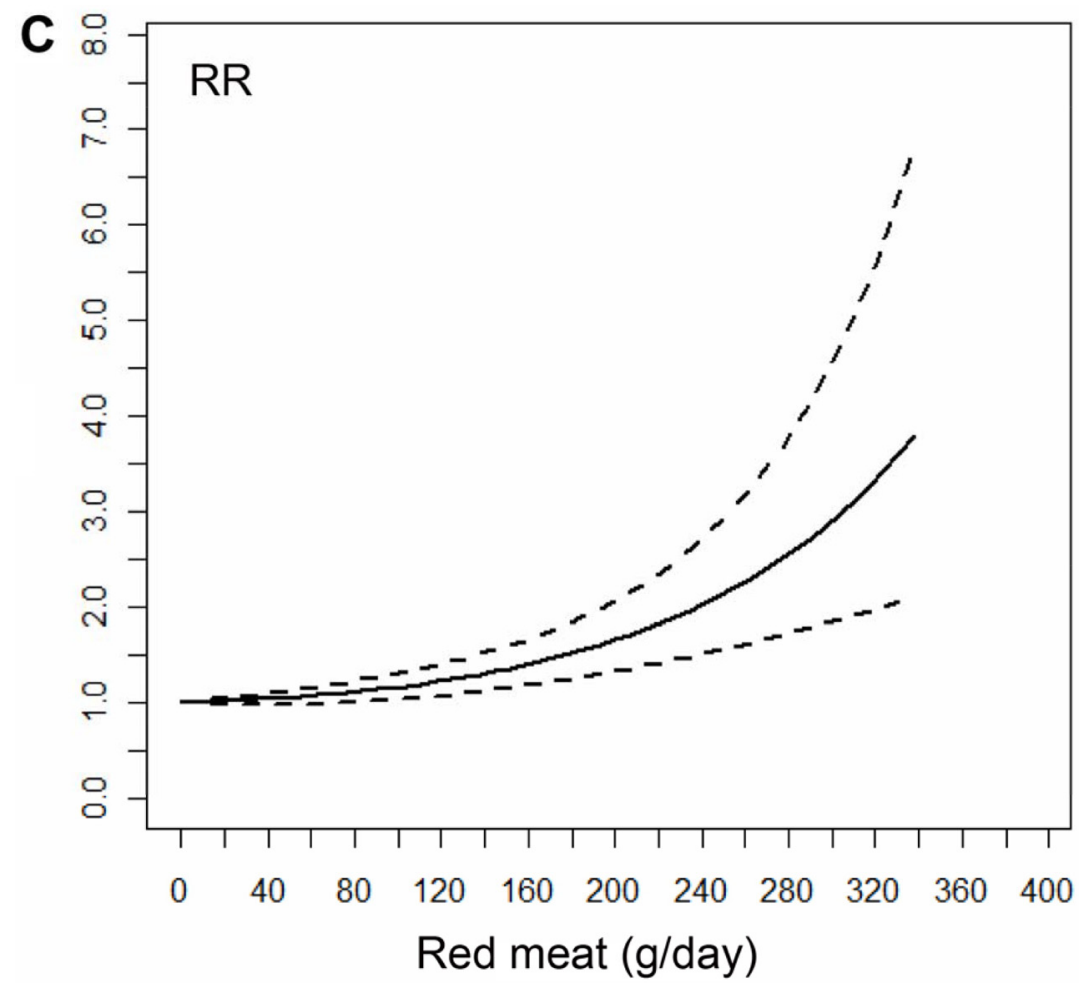

Figure 2: The summary risk association between red meat intake and risk of renal cell carcinoma according to (A) the highest vs. lowest analysis; (B) linear dose-response analysis (Per $100 \mathrm{~g} /$ day increment); (C) non-linear dose-response analysis. Studies are sub-grouped according to design.

Egger's test $(P=0.145)$ and Begg's test $(P=0.183)$, did not indicate publication bias. The trim-and-fill method indicated that two additional risk estimates were needed to balance the funnel plot (Figure 4B), and the summary risk estimates were unchanged $(\mathrm{SRR}=1.12$; 95\% CI, 1.02-1.23).

\section{Individual meat items}

There were positive associations between RCC risk with the consumption of beef $(\mathrm{SRR}=1.89 ; 95 \% \mathrm{CI}$, $1.25-2.86)$, hamburger (SRR $=1.41 ; 95 \% \mathrm{CI}, 1.12-1.78)$, and $\mathrm{ham} / \mathrm{salami} / \mathrm{bacon} / \mathrm{sausage}(\mathrm{SRR}=1.30 ; 95 \% \mathrm{CI}$, 1.16-1.47). RCC risk was not positively associated with the intake of pork or barbecued/pan-fried/broiled meat (Supplementary Table 2).

\section{DISCUSSION}

The results of this comprehensive meta-analysis show that the consumption of red and processed meat is associated with increased RCC risk, as per the high vs. low and linear dose-response meta-analyses. There was significant heterogeneity across studies for both red and processed meat intake. In non-linear models, RCC risk appeared to increase approximately linearly with increased intake of processed meat, whereas there was evidence of non-linear increased risk with increased intake of red meat. Among individual red and processed meat types, there were statistically significant positive associations for the intake of beef, salami/ham/bacon/sausage, and hamburger.

Several mechanisms have been proposed to explain how the consumption of red and processed meat enhances cancer risk, and include the high intake of proteins and fats and intake of carcinogens (e.g., NOCs, HCAs, PAHs) $[49,50]$. A large prospective cohort study observed increased risk of RCC with high consumption of nitrate and nitrite, the precursor of NOCs, and total RCC (hazard ratio $=1.28,95 \%$ CI, 1.10-1.49) [51]. In animal studies, benzo (a) pyrene $(\mathrm{BaP})$ and PhIP were two of the most potent PAHs [52]. Epidemiological studies have found a positive association between $\mathrm{BaP}$ and $\mathrm{PhIP}$ and $\mathrm{RCC}$ $[34,36]$. The high saturated fat content of red and processed meat has also been proposed as a culprit for the increased risk of RCC in some studies [53], but not in other studies [54, 55].

In comparison with previous meta-analyses [29, 30], the present updated analysis included an additional 11 studies (two updated studies), and a total 14,285 patients with RCC and 1,821,615 controls/participants, which can provide sufficient power for detecting the putative moderate associations. In addition, we conducted comprehensive analyses based on high vs. low, linear, and non-linear dose-response models; importantly, we 
Table 3: Subgroup analyses of red and processed meat intake and renal cell carcinoma risk, high vs. low

\begin{tabular}{|c|c|c|c|c|c|c|c|c|c|c|}
\hline \multirow[b]{2}{*}{ Sub-groups } & \multicolumn{5}{|c|}{ Red meat } & \multicolumn{5}{|c|}{ Processed meat } \\
\hline & $\begin{array}{c}\text { Studies, } \\
\mathbf{n}\end{array}$ & SRR $(95 \%$ CI $)$ & $\begin{array}{c}P \text { for } \\
\text { heterogeneity }\end{array}$ & $I^{2}(\%)$ & $\begin{array}{c}P \text { for } \\
\text { difference }\end{array}$ & $\begin{array}{c}\text { Studies, } \\
n\end{array}$ & SRR $(95 \%$ CI $)$ & $\begin{array}{c}P \text { for } \\
\text { heterogeneity }\end{array}$ & $I^{2}(\%)$ & $\begin{array}{c}P \text { for } \\
\text { difference }\end{array}$ \\
\hline All & 19 & $1.36(1.16-1.58)$ & $<0.001$ & 71.3 & & 19 & $1.13(1.03-1.24)$ & 0.014 & 45.6 & \\
\hline Design & & & & & 0.751 & & & & & 0.956 \\
\hline Cohort & 5 & $1.07(0.96-1.19)$ & 0.398 & 1.5 & & 4 & $1.11(0.99-1.25)$ & 0.797 & 0 & \\
\hline Case-control & 14 & $1.46(1.18-1.81)$ & $<0.001$ & 75.0 & & 14 & $1.13(1.00-1.27)$ & 0.004 & 55.4 & \\
\hline Sources of control & & & & & 0.470 & & & & & 0.152 \\
\hline Population-based & 8 & $1.29(1.06-1.58)$ & 0.021 & 57.4 & & 9 & $1.18(1.04-1.34)$ & 0.024 & 54.5 & \\
\hline Hospital-based & 6 & $1.75(1.10-2.78)$ & $<0.001$ & 84.7 & & 6 & $1.03(0.79-1.34)$ & 0.059 & 50.6 & \\
\hline $\begin{array}{c}\text { Geographic } \\
\text { locations }\end{array}$ & & & & & 0.038 & & & & & 0.178 \\
\hline Europe & 6 & $1.04(0.86-1.26)$ & 0.069 & 51.2 & & 5 & $0.98(0.78-1.23)$ & 0.065 & 54.7 & \\
\hline USA & 10 & $1.44(1.17-1.76)$ & 0.001 & 68.6 & & 9 & $1.20(1.07-1.34)$ & 0.062 & 46.2 & \\
\hline South America & 2 & $3.12(1.87-5.20)$ & $<0.001$ & 72.3 & & 3 & $1.16(0.81-1.67)$ & 0.275 & 22.7 & \\
\hline Asia & 1 & $1.30(0.69-2.460$ & - & & & 1 & $1.60(0.58-4.44)$ & - & & \\
\hline Data available & & & & & 0.189 & & & & & 0.424 \\
\hline Self-administered & 12 & $1.25(1.07-1.45)$ & 0.002 & 63.5 & & 8 & $1.17(1.03-1.33)$ & 0.069 & 46.7 & \\
\hline Interview & 7 & $1.79(1.16-2.75)$ & $<0.001$ & 78.8 & & 11 & $1.09(0.95-1.26)$ & 0.052 & 43.7 & \\
\hline Type of FFQ & & & & & 0.294 & & & & & 0.857 \\
\hline Validated & 16 & $1.44(1.20-1.75)$ & $<0.001$ & 75.8 & & 15 & $1.12(1.00-1.25)$ & 0.012 & 49.9 & \\
\hline Not available & 4 & $1.10(0.90-1.33)$ & 0.317 & 15.0 & & 4 & $1.17(0.94-1.44)$ & 0.172 & 40.0 & \\
\hline \multicolumn{11}{|l|}{ Study quality score } \\
\hline $\begin{array}{c}\text { High } \\
(\text { NOS score }>6)\end{array}$ & 16 & $1.32(1.30-1.54)$ & $<0.001$ & 71.5 & 0.464 & 17 & $1.13(1.03-1.25)$ & 0.011 & 48.4 & 0.713 \\
\hline $\begin{array}{c}\text { Low } \\
(\text { NOS score } \leq 6)\end{array}$ & 3 & $1.78(0.79-4.00)$ & 0.012 & 77.4 & & 2 & $1.03(0.66-1.63)$ & 0.177 & 45.1 & \\
\hline \multicolumn{11}{|l|}{ Adjustments } \\
\hline BMI, yes & 15 & $1.39(1.16-1.66)$ & $<0.001$ & 77.4 & 0.705 & 17 & $1.12(1.01-1.24)$ & 0.008 & 50.2 & 0.426 \\
\hline no & 4 & $1.22(0.95-1.57)$ & 0.842 & 0 & & 2 & $1.34(0.91-1.98)$ & 0.713 & 0 & \\
\hline Smoking, yes & 15 & $1.30(1.11-1.52)$ & $<0.001$ & 73.1 & 0.302 & 15 & $1.12(1.01-1.25)$ & 0.006 & 53.4 & 0.979 \\
\hline no & 4 & $1.75(1.10-2.79)$ & 0.130 & 47.0 & & 4 & $1.17(0.92-1.50)$ & 0.441 & 0 & \\
\hline Energy intake, yes & 8 & $1.21(1.01-1.46)$ & $<0.001$ & 77.9 & 0.167 & 10 & $1.11(1.01-1.26)$ & 0.001 & 65.2 & 0.716 \\
\hline no & 11 & $1.49(1.15-1.93)$ & 0.001 & 68.0 & & 9 & $1.17(1.01-1.35)$ & 0.637 & 0 & \\
\hline Hypertension, yes & 7 & $1.35(1.07-1.70)$ & $<0.001$ & 78.7 & 0.929 & 7 & $1.09(1.00-1.18)$ & 0.457 & 0 & 0.947 \\
\hline no & 12 & $1.38(1.10-1.73)$ & $<0.001$ & 68.2 & & 12 & $1.13(0.97-1.32)$ & 0.009 & 54.6 & \\
\hline $\begin{array}{l}\text { Consumption of } \\
\text { vegetables and } \\
\text { fruits, yes }\end{array}$ & 7 & $1.40(1.07-1.85)$ & $<0.001$ & 79.2 & 0.883 & 7 & $1.17(1.03-1.34)$ & 0.152 & 34.6 & 0.368 \\
\hline No & 12 & $1.33(1.09-1.63)$ & $<0.001$ & 67.4 & & 12 & $1.08(0.94-1.24)$ & 0.012 & 54.5 & \\
\hline Alcohol, yes & 10 & $1.17(1.02-1.34)$ & 0.030 & 51.2 & 0.151 & 11 & $1.15(1.01-1.30)$ & 0.002 & 62.4 & 0.635 \\
\hline No & 9 & $1.65(1.18-2.31)$ & $<0.001$ & 77.2 & & 8 & $1.08(0.94-1.24)$ & 0.684 & 0 & \\
\hline
\end{tabular}

performed rigorous quality assessment. We also explored the association between specific subtypes of meat and RCC risk. Finally, by conducting a meta-regression analysis, we could explore the source of heterogeneity between studies.

We found that red and processed meat consumption was significantly associated with increased risk of RCC in the case-control studies, which might drive the overall epidemiological findings of the present study, but not in the cohort studies. Case-control studies are more susceptible to recall and selection bias than are cohort studies, as lifestyles and diet habits in retrospective case-control studies are determined after the diagnosis of cancer. Although the meta-regression results suggested that study design did not significantly alter the aforementioned associations, we observed that the positive association 
was weaker in the cohort studies than in the case-control studies. Therefore, the finding that red and processed meat consumption is associated with increased RCC risk should be received with caution.

The present meta-analysis has several limitations. First, inaccurate assessments of dietary intake could have led to overestimations of the range of intakes and consequent underestimation of the magnitude of the aforementioned relationship [56, 57]. Not all studies used validated semiquantitative FFQs for dietary assessment; however, subgroup analyses showed that the use of validated vs. non-validated FFQs did not significantly

\section{Processed meat}

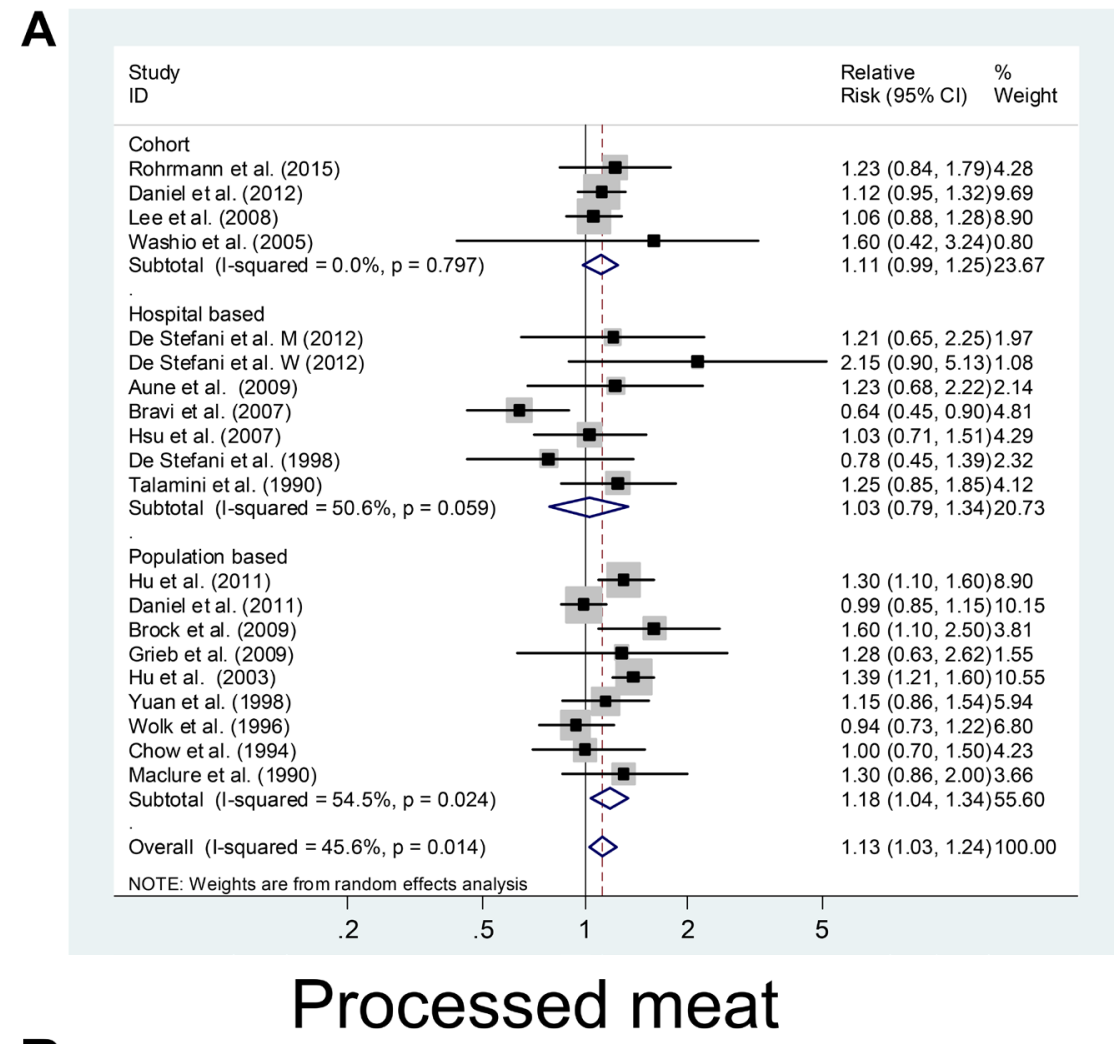

B

Study
ID




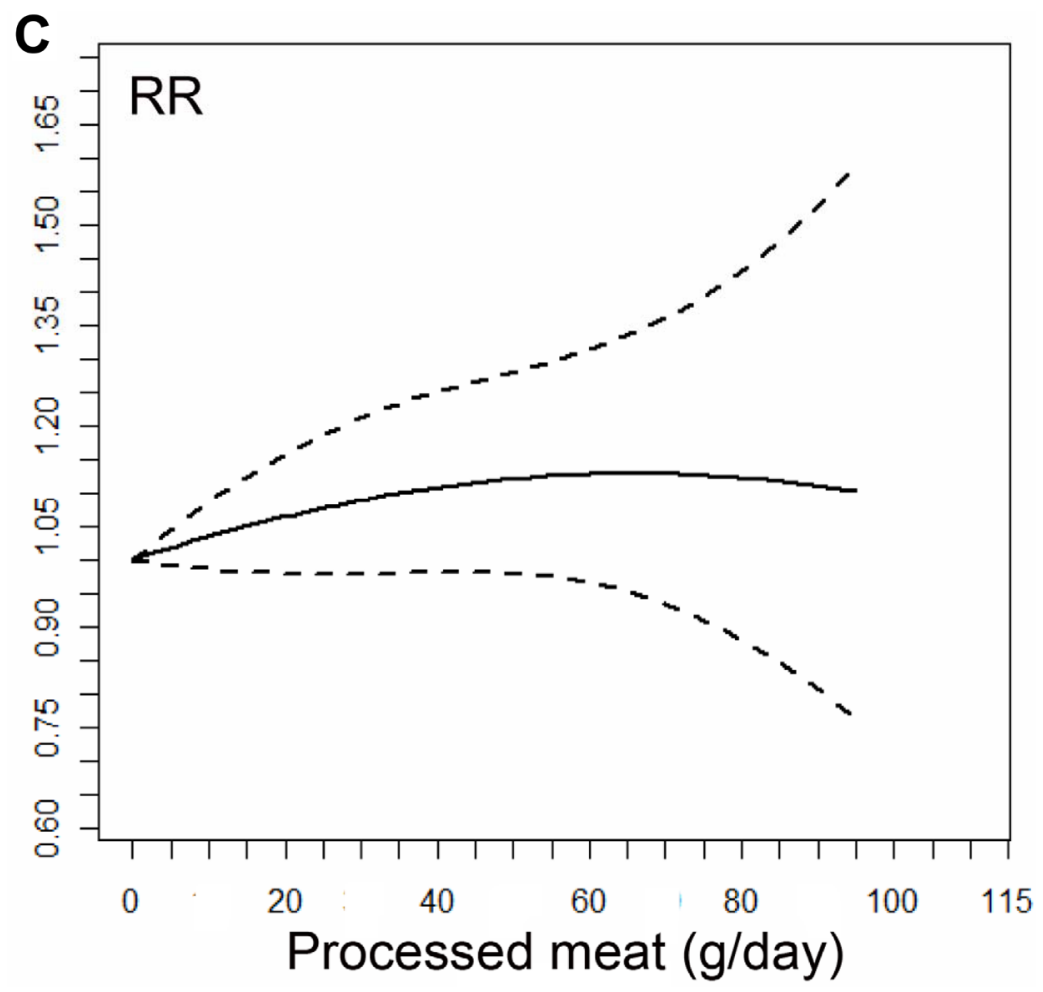

Figure 3: The summary risk association between processed meat intake and risk of renal cell carcinoma according to (A) the highest vs. lowest analysis; (B) linear dose-response analysis (Per $50 \mathrm{~g} /$ day increment); (C) non-linear dose-response analysis. Studies are sub-grouped according to design.

affect the association between the consumption of red and processed meat and RCC risk. Although some FFQs were not validated, its reproducibility has been confirmed, with the correlation coefficients between the two assessments being 0.77 and 0.55 for red meat and processed meat, respectively [58]. In addition, analyses of the highest vs. lowest intake are limited because they do not account for true differences among studies. For example, the definition of lowest intake of red meat ranged from 0 to $<1$ time/month [16], and the highest intake ranged from 1 time/week [16] to $>365 \mathrm{~g} /$ day [23].

Second, there was great inter-study heterogeneity. Stratified and meta-regression analyses revealed a significant positive association between studies from North America (but not from Europe), and study location was the only significant factor in the association between intake of red meat and RCC risk. This might be attributed to the fact that different populations consume different types, levels of meat, and their cooking practices differ, which may partly explain the high heterogeneity among the included studies. Additionally, there was considerable heterogeneity in the dose-response analysis models, which might be ascribed to a consequence of the conversions of the intake units.

Third, the residual confounders inherent in primary observational studies are always of concern. Although most of the included studies reported adjusted risk estimates of RCC for confounders, some appeared to have failed to fully control for confounders. For example, only seven studies used adjustments for history of hypertension, which is one of the established risk factors of RCC [7]. High intake of red meat and processed meat is likely to be associated with other unhealthy lifestyle choices, for example, smoking, obesity, and lower intake of vegetables and fruits, all of which are indicated as risk factors for RCC $[5,6]$. In addition, alcohol consumption is common in people with high intake of red and processed meat, and moderate alcohol consumption was identified as a protective factor against RCC [59]. When we limited the meta-analysis to studies controlled for BMI, smoking, alcohol use, and intake of vegetables and fruits, the aforementioned positive associations were not significantly modified.

Fourth, HCA and PAH formation increases with cooking temperature and duration; however, data on the degree of meat doneness in the included studies were not available. Additionally, the non-linear trend with intake of red meat should be interpreted with caution due to the low statistical power in the extremes of red meat intake distribution. This is an issue of the fractional polynomial method. Most of the included studies were based on data from Western populations; additional research in other populations is warranted to generalize these findings.

Lastly, we acknowledge the presence of significant publication bias in the results for red meat intake. The overall risk estimates for the association for red meat consumption were probably an overestimation, as small studies with null results tend not to be published. Indeed, 
the trim-and-fill method indicated that eight additional risk estimates were needed to balance the funnel plot, and the summary risk estimates were attenuated and not statistically significant.

In conclusion, our limited data suggest that high intake of red and processed meat may increase RCC risk. However, because the effect was only found in case-control studies and might be a consequence of bias, confounding factors, and importantly, publication bias, further prospective epidemiological studies that control for possible confounders and that examine the association between meat consumption and RCC risk are required.

\section{A Filled funnel plot with pseudo $95 \%$ confidence limits}

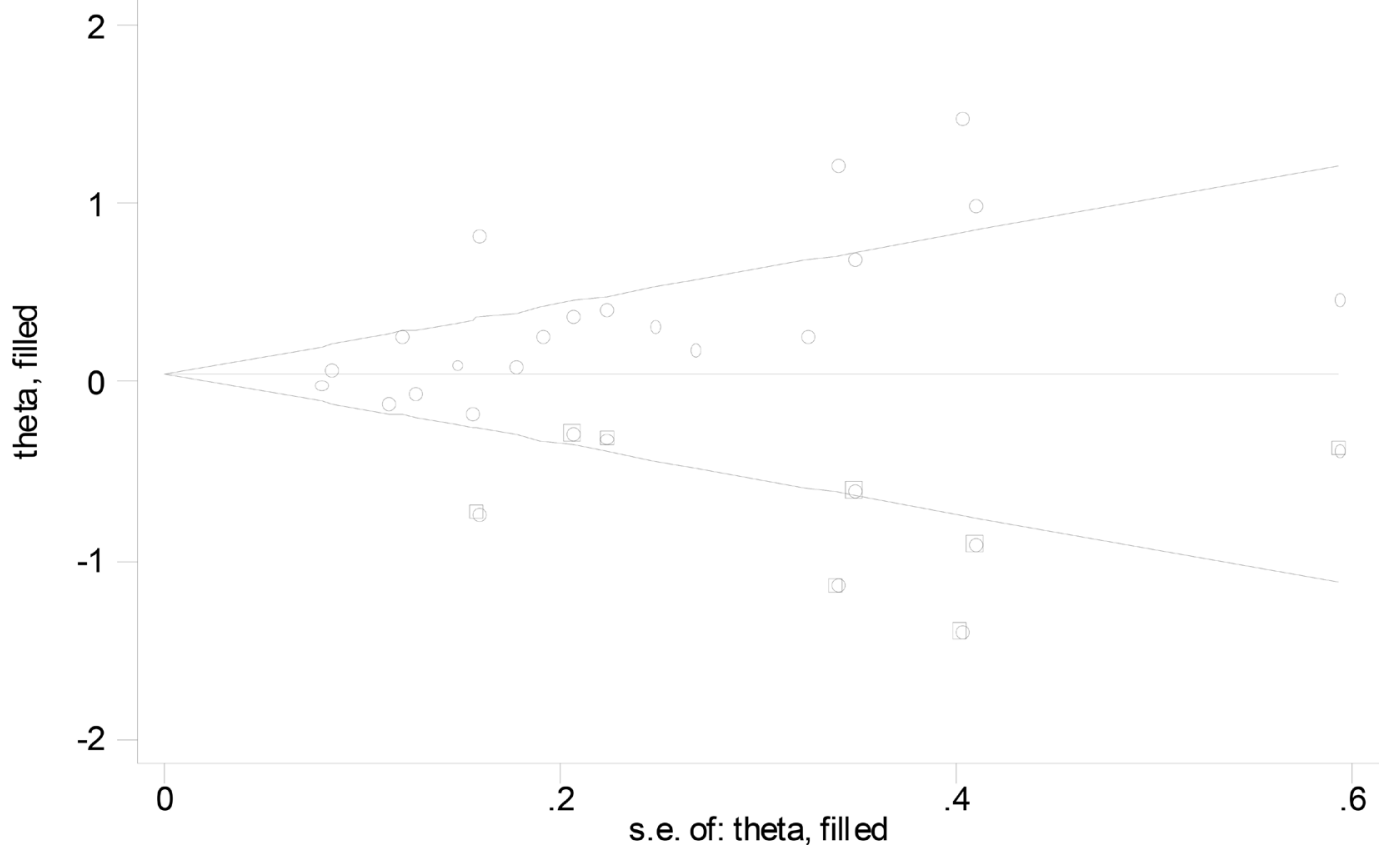

B Filled funnel plot with pseudo $95 \%$ confidence limits

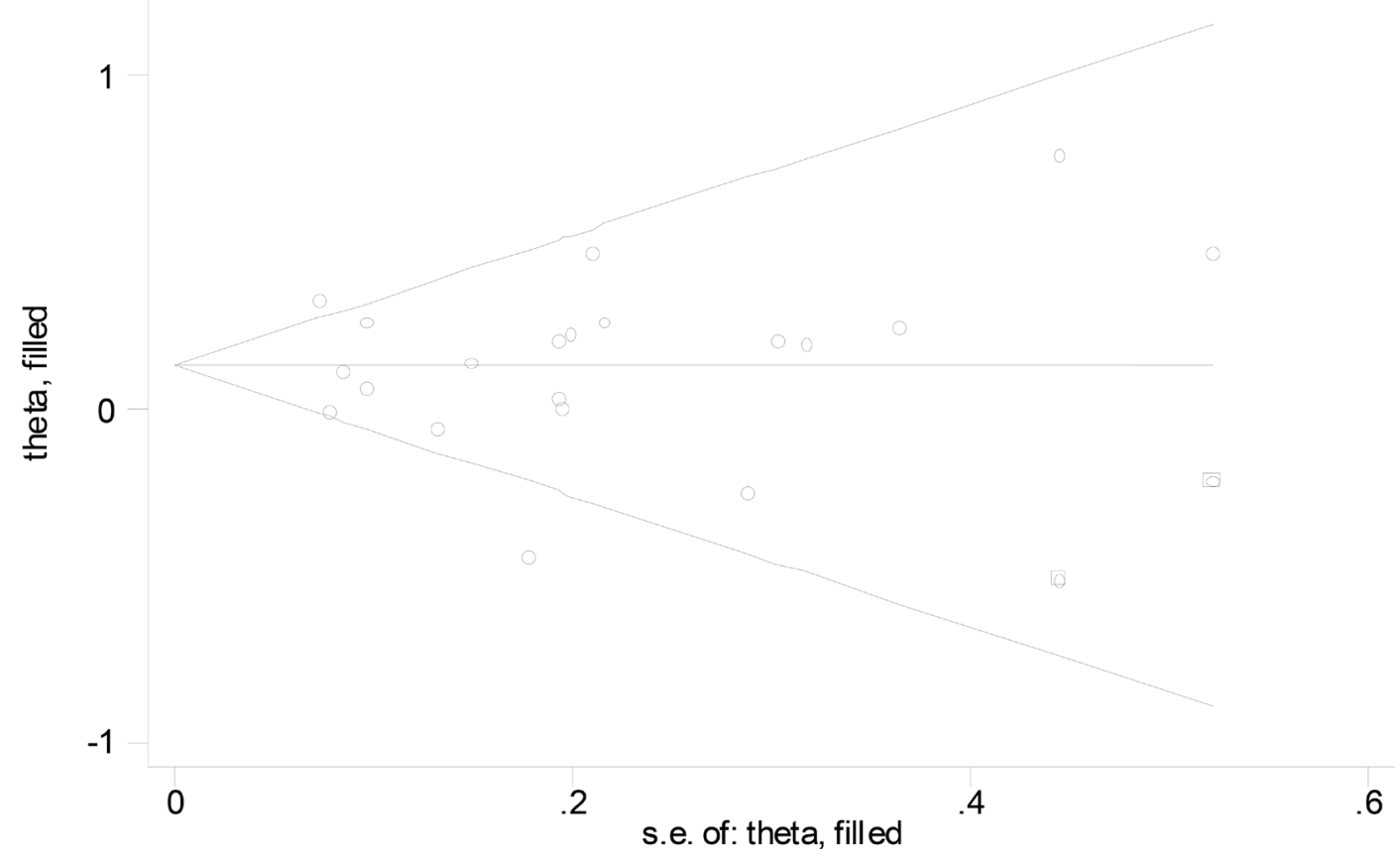

Figure 4: Filled funnel plot of log relative risk vs. standard error of log relative risks in studies that evaluated the effect of red meat (A) and processed meat (B) intake on the risk of renal cell carcinoma. 


\section{ACKNOWLEDGMENTS}

Shaojing Zhang, Juanjuan He and Qingwei Wang participated in the design of this manuscript. Shaojing Zhang, Juanjuan He participated in abstracting the data and performing statistical analysis. All authors read and approved the final manuscript.

\section{CONFLICTS OF INTEREST}

None.

\section{REFERENCES}

1. Siegel RL, Miller KD, Jemal A. Cancer statistics, 2015. CA Cancer J Clin. 2015; 65:5-29.

2. Ljungberg B, Campbell SC, Choi HY, Jacqmin D, Lee JE, Weikert S, Kiemeney LA. The epidemiology of renal cell carcinoma. Eur Urol. 2011; 60:615-21.

3. Ferlay JS, Bray F, Forman D, Mathers C, Parkin DM. GLOBOCAN 2008 v1.2 Cancer Incidence and Mortality Worldwide: IARC CancerBase No. 10. Vol. 2012. Internation Agency for Research on Cancer; Lyon, France. 2008.

4. Znaor A, Lortet-Tieulent J, Laversanne M, Jemal A, Bray F. International variations and trends in renal cell carcinoma incidence and mortality. Eur Urol. 2015; 67:519-30.

5. Hunt JD, van der Hel OL, McMillan GP, Boffetta P, Brennan P. Renal cell carcinoma in relation to cigarette smoking: meta-analysis of 24 studies. Int J Cancer. 2005; 114:101-8.

6. Wang F, Xu Y. Body mass index and risk of renal cell cancer: a dose-response meta-analysis of published cohort studies. Int J Cancer. 2014; 135:1673-86.

7. Corrao G, Scotti L, Bagnardi V, Sega R. Hypertension, antihypertensive therapy and renal-cell cancer: a metaanalysis. Curr Drug Saf. 2007; 2:125-33.

8. Lee HH, Choi KH, Yang SC, Han WK. Renal cell carcinoma in kidney transplant recipients and dialysis patients. Korean J Urol. 2012; 53:229-33.

9. Clague J, Lin J, Cassidy A, Matin S, Tannir NM, Tamboli P, Wood CG, Wu X. Family history and risk of renal cell carcinoma: results from a case-control study and systematic meta-analysis. Cancer Epidemiol Biomarkers Prev. 2009; 18:801-7.

10. Choueiri TK, Je Y, Cho E. Analgesic use and the risk of kidney cancer: a meta-analysis of epidemiologic studies. Int J Cancer. 2016; 134:384-96.

11. Larsson SC, Wolk A. Diabetes mellitus and incidence of kidney cancer: a meta-analysis of cohort studies. Diabetologia. 2011; 54:1013-8.

12. Kabaria R, Klaassen Z, Terris MK. Renal cell carcinoma: links and risks. Int J Nephrol Renovasc Dis. 2016; 9:45-52.

13. Ghoshal A, Preisegger KH, Takayama S, Thorgeirsson SS, Snyderwine EG. Induction of mammary tumors in female
Sprague-Dawley rats by the food-derived carcinogen 2-amino-1-methyl-6-phenylimidazo[4,5-b]pyridine and effect of dietary fat. Carcinogenesis. 1994; 15:2429-33.

14. Rohrmann S, Hermann S, Linseisen J. Heterocyclic aromatic amine intake increases colorectal adenoma risk: findings from a prospective European cohort study. Am J Clin Nutr. 2009; 89:1418-24.

15. Lee JE, Spiegelman D, Hunter DJ, Albanes D, Bernstein L, van den Brandt PA, Buring JE, Cho E, English DR, Freudenheim JL, Giles GG, Graham S, Horn-Ross PL, et al. Fat, protein, and meat consumption and renal cell cancer risk: a pooled analysis of 13 prospective studies. J Natl Cancer Inst. 2008; 100:1695-706.

16. Hsu CC, Chow WH, Boffetta P, Moore L, Zaridze D, Moukeria A, Janout V, Kollarova H, Bencko V, Navratilova M, Szeszenia-Dabrowska N, Mates D, Brennan P. Dietary risk factors for kidney cancer in Eastern and Central Europe. Am J Epidemiol. 2007; 166:62-70.

17. Bravi F, Bosetti C, Scotti L, Talamini R, Montella M, Ramazzotti V, Negri E, Franceschi S, La Vecchia C. Food groups and renal cell carcinoma: a case-control study from Italy. Int J Cancer. 2007; 120:681-5.

18. Washio M, Mori M, Sakauchi F, Watanabe Y, Ozasa K, Hayashi K, Miki T, Nakao M, Mikami K, Ito Y, Wakai K, Tamakoshi A, and JACC Study Group. Risk factors for kidney cancer in a Japanese population: findings from the JACC Study. J Epidemiol. 2005 (Suppl 2); 15:S203-11.

19. Hu J, Mao Y, White K, and Canadian Cancer Registries Epidemiology Research Group. Diet and vitamin or mineral supplements and risk of renal cell carcinoma in Canada. Cancer Causes Control. 2003; 14:705-14.

20. Handa K, Kreiger N. Diet patterns and the risk of renal cell carcinoma. Public Health Nutr. 2002; 5:757-67.

21. Tavani A, La Vecchia C, Gallus S, Lagiou P, Trichopoulos D, Levi F, Negri E. Red meat intake and cancer risk: a study in Italy. Int J Cancer. 2000; 86:425-8.

22. Yuan JM, Gago-Dominguez M, Castelao JE, Hankin JH, Ross RK, Yu MC. Cruciferous vegetables in relation to renal cell carcinoma. Int J Cancer. 1998; 77:211-6.

23. De Stefani E, Fierro L, Mendilaharsu M, Ronco A, Larrinaga MT, Balbi JC, Alonso S, Deneo-Pellegrini H. Meat intake, 'mate' drinking and renal cell cancer in Uruguay: a case-control study. Br J Cancer. 1998; 78:1239-43.

24. Wolk A, Gridley G, Niwa S, Lindblad P, McCredie M, Mellemgaard A, Mandel JS, Wahrendorf J, McLaughlin JK, Adami HO. International renal cell cancer study. VII. Role of diet. Int J Cancer. 1996; 65:67-73.

25. Chow WH, Gridley G, McLaughlin JK, Mandel JS, Wacholder S, Blot WJ, Niwa S, Fraumeni JF, Jr. Protein intake and risk of renal cell cancer. J Natl Cancer Inst. 1994; 86:1131-9.

26. Talamini R, Baron AE, Barra S, Bidoli E, La Vecchia C, Negri E, Serraino D, Franceschi S. A case-control study of risk factor for renal cell cancer in northern Italy. Cancer Causes Control. 1990; 1:125-31. 
27. Maclure M, Willett W. A case-control study of diet and risk of renal adenocarcinoma. Epidemiology. 1990; 1:430-40.

28. Fraser GE, Phillips RL, Beeson WL. Hypertension, antihypertensive medication and risk of renal carcinoma in California Seventh-Day Adventists. Int J Epidemiol. 1990; 19:832-8.

29. Alexander DD, Cushing CA. Quantitative assessment of red meat or processed meat consumption and kidney cancer. Cancer Detect Prev. 2009; 32:340-51.

30. Faramawi MF, Johnson E, Fry MW, Sall M, Zhou Y. Consumption of different types of meat and the risk of renal cancer: meta-analysis of case-control studies. Cancer Causes Control. 2007; 18:125-33.

31. Melkonian SC, Daniel CR, Ye Y, Tannir NM, Karam JA, Matin SF, Wood CG, Wu X. Gene-environment interaction of genome-wide association study-identified susceptibility loci and meat-cooking mutagens in the etiology of renal cell carcinoma. Cancer. 2016; 122:108-15.

32. Rohrmann S, Linseisen J, Overvad K, Lund Wurtz AM, Roswall N, Tjonneland A, Boutron-Ruault MC, Racine A, Bastide N, Palli D, Agnoli C, Panico S, Tumino R, et al. Meat and fish consumption and the risk of renal cell carcinoma in the European prospective investigation into cancer and nutrition. Int J Cancer. 2015; 136: E423-31.

33. De Stefani E, Boffetta P, Ronco AL, Deneo-Pellegrini H, Correa P, Acosta G, Mendilaharsu M, Luaces ME, Silva C. Processed meat consumption and risk of cancer: a multisite case-control study in Uruguay. Br J Cancer. 2012; 107:1584-8.

34. Daniel CR, Cross AJ, Graubard BI, Park Y, Ward MH, Rothman N, Hollenbeck AR, Chow WH, Sinha R. Large prospective investigation of meat intake, related mutagens, and risk of renal cell carcinoma. Am J Clin Nutr. 2012; 95:155-62.

35. Hu J, La Vecchia C, Morrison H, Negri E, Mery L, and Canadian Cancer Registries Epidemiology Research Group. Salt, processed meat and the risk of cancer. Eur J Cancer Prev. 2011; 20:132-9.

36. Daniel CR, Schwartz KL, Colt JS, Dong LM, Ruterbusch JJ, Purdue MP, Cross AJ, Rothman N, Davis FG, Wacholder S, Graubard BI, Chow WH, Sinha R. Meat-cooking mutagens and risk of renal cell carcinoma. Br J Cancer. 2011; 105:1096-104.

37. Grieb SM, Theis RP, Burr D, Benardot D, Siddiqui T, Asal NR. Food groups and renal cell carcinoma: results from a case-control study. J Am Diet Assoc. 2009; 109:656-67.

38. De Stefani E, Aune D, Boffetta P, Deneo-Pellegrini H, Ronco AL, Acosta G, Brennan P, Ferro G, Mendilaharsu M. Salted meat consumption and the risk of cancer: a multisite case-control study in Uruguay. Asian Pac J Cancer Prev. 2009; 10:853-7.

39. Brock KE, Gridley G, Chiu BC, Ershow AG, Lynch CF, Cantor KP. Dietary fat and risk of renal cell carcinoma in the USA: a case-control study. Br J Nutr. 2009; 101:1228-38.

40. Aune D, De Stefani E, Ronco A, Boffetta P, DeneoPellegrini H, Acosta G, Mendilaharsu M. Meat consumption and cancer risk: a case-control study in Uruguay. Asian Pac J Cancer Prev. 2009; 10:429-36.

41. Stroup DF, Berlin JA, Morton SC, Olkin I, Williamson GD, Rennie D, Moher D, Becker BJ, Sipe TA, Thacker SB. Meta-analysis of observational studies in epidemiology: a proposal for reporting. Meta-analysis Of Observational Studies in Epidemiology (MOOSE) group. JAMA. 2000; 283:2008-12.

42. Wells GA, O'Connell D, Peterson J, Welch V, Losos M, Tugwell P. The Newcastle-Ottawa Scale (NOS) for assessing the quality of nonrandomised studies in metaanalyses. Available at: http://www.ohri.ca/programs/clinical_ epidemiology/oxford.asp. Accessed June 15, 2012.

43. DerSimonian R, Laird N. Meta-analysis in clinical trials. Control Clin Trials. 1986; 7:177-88.

44. Higgins JP, Thompson SG, Deeks JJ, Altman DG. Measuring inconsistency in meta-analyses. BMJ. 2003; 327:557-60.

45. Greenland S, Longnecker MP. Methods for trend estimation from summarized dose-response data, with applications to meta-analysis. Am J Epidemiol. 1992; 135:1301-9.

46. Orsini NB, Greenland S. Generalized least squares for trend estimation of summarized dose-response data. Stata J. 2006; $6: 40-57$.

47. Royston P. A strategy for modelling the effect of a continuous covariate in medicine and epidemiology. Stat Med. 2000; 19:1831-47.

48. Di Maso M, Talamini R, Bosetti C, Montella M, Zucchetto A, Libra M, Negri E, Levi F, La Vecchia C, Franceschi S, Serraino D, Polesel J. Red meat and cancer risk in a network of case-control studies focusing on cooking practices. Ann Oncol. 2013; 24:3107-12.

49. Whiteman DC, Wilson LF. The fractions of cancer attributable to modifiable factors: A global review. Cancer Epidemiol. 2016; 44:203-21.

50. Ito $\mathrm{N}$, Hasegawa $\mathrm{R}$, Sano $\mathrm{M}$, Tamano S, Esumi H, Takayama S, Sugimura T. A new colon and mammary carcinogen in cooked food, 2-amino-1-methyl-6phenylimidazo[4,5-b]pyridine (PhIP). Carcinogenesis. $1991 ; 12: 1503-6$.

51. Dellavalle CT, Daniel CR, Aschebrook-Kilfoy B, Hollenbeck AR, Cross AJ, Sinha R, Ward MH. Dietary intake of nitrate and nitrite and risk of renal cell carcinoma in the NIH-AARP Diet and Health Study. Br J Cancer. 2013; 108:205-12.

52. Culp SJ, Gaylor DW, Sheldon WG, Goldstein LS, Beland FA. A comparison of the tumors induced by coal tar and benzo[a]pyrene in a 2-year bioassay. Carcinogenesis. 1998; 19:117-24.

53. Hu J, La Vecchia C, DesMeules M, Negri E, Mery L, and Canadian Cancer Registries Epidemiology Research Group. Nutrient and fiber intake and risk of renal cell carcinoma. Nutr Cancer. 2008; 60:720-8.

54. Allen NE, Balkwill A, Beral V, Green J, Reeves G, and Million Women Study Collaborators. Fluid intake and 
incidence of renal cell carcinoma in UK women. Br J Cancer. 2011; 104:1487-92.

55. Allen NE, Roddam AW, Sieri S, Boeing H, Jakobsen MU, Overvad K, Tjonneland A, Halkjaer J, Vineis P, Contiero P, Palli D, Tumino R, Mattiello A, et al. A prospective analysis of the association between macronutrient intake and renal cell carcinoma in the European Prospective Investigation into Cancer and Nutrition. Int J Cancer. 2009; 125:982-7.

56. Prentice RL. Dietary assessment and the reliability of nutritional epidemiology reports. Lancet. 2003; 362:182-3.

57. Willett WC, Sampson L, Stampfer MJ, Rosner B, Bain C, Witschi J, Hennekens CH, Speizer FE. Reproducibility and validity of a semiquantitative food frequency questionnaire. Am J Epidemiol. 1985; 122:51-65.

58. Ronco AL, De Stefani E, Boffetta P, Deneo-Pellegrini H, Acosta G, Mendilaharsu M. Food patterns and risk of breast cancer: A factor analysis study in Uruguay. Int J Cancer. 2006; 119:1672-8.

59. Bellocco R, Pasquali E, Rota M, Bagnardi V, Tramacere I, Scotti L, Pelucchi C, Boffetta P, Corrao G, La Vecchia C. Alcohol drinking and risk of renal cell carcinoma: results of a meta-analysis. Ann Oncol. 2012; 23:2235-44. 